\title{
Spectroscopic Properties of Colloidal Indium Phosphide Quantum Wires
}

Fudong Wang, ${ }^{\S \ddagger}$ Heng Yu,${ }^{\$ \dagger}$ Jingbo Li, ${ }^{\natural \ddagger}$ Qingling Hang, ${ }^{\&}$ Dmitry Zemlyanov, ${ }^{\&}$ Patrick C. Gibbons, ${ }^{\# \neq}$ Lin-Wang Wang, ${ }^{,}$David B. Janes, ${ }^{,}$and William E. Buhro*\$*

Department of Chemistry and Physics and Center for Materials Innovation, Washington

University, St. Louis, MO 63130-4899; Lawrence Berkeley National Laboratory, 1 Cyclotron

Road, Berkeley, CA 94720; and Birck Nanotechnology Center, Purdue University, West

Lafayette, IN 47907.

Received June 04, 2007. E-mail: buhro@,wustl.edu.

Title running head: Spectroscopic Properties of InP Quantum Wires

Abstract: Colloidal InP quantum wires are grown by the solution-liquid-solid (SLS) method, and passivated with the traditional quantum dots surfactants 1-hexadecylamine and tri-n-octylphosphine oxide. The size dependence of the band gaps in the wires are determined from the absorption spectra, and compared to other experimental results for InP quantum dots and wires, and to the predictions of theory. The photoluminescence behavior of the wires is also investigated. Efforts to enhance photoluminescence efficiencies through photochemical etching in the presence of HF result only in photochemical thinning or photo-oxidation, without a significant influence on quantum-wire photoluminescence. However, photo-oxidation produces residual dot and rod domains within the wires, which are luminescent. The results establish that the quantum-wire band gaps are weakly influenced by the nature of the surface passivation, and that colloidal quantum wires have intrinsically low photoluminescence efficiencies.

\footnotetext{
${ }^{\dagger}$ Current address: Stanford Genome Technology Center, Department of Biochemistry, Stanford University, 855 California Ave, Palo Alto, CA 94304.

${ }^{3}$ Current address: State Key Laboratory for Superlattice and Microstructure, Institute of Semiconductor, Chinese Academy of Science, P. O. Box 912, Beijing 100083, China.

${ }^{\S}$ Department of Chemistry, Washington University

\#Department of Physics, Washington University

Center for Materials Innovation, Washington University

${ }^{t}$ Lawrence Berkeley National Laboratory

${ }^{\&}$ Birck Nanotechnology Center, Purdue University
} 


\section{Introduction}

The motivation for this study was to determine if InP quantum wires having optimal surface passivation would exhibit the well-resolved absorption and emission spectra, and high

photoluminescence (PL) quantum yields, that have been achieved with InP quantum dots. ${ }^{1,2}$ Therefore, a detailed analysis of the band gaps and PL behavior exhibited by InP quantum wires is provided. We describe efforts to enhance quantum-wire PL by photochemical etching with HF, which has proven successful for InP quantum dots. ${ }^{1,2}$ We show that with InP quantum wires the method affords only photochemical wire thinning or photo-oxidation. The PL enhancements ultimately obtained are due to residual quantum-dot and quantum-rod domains left in the wires after photo-oxidation. The results highlight the intrinsic difficulties in obtaining efficient PL from colloidal semiconductor quantum wires.

Additionally, the InP quantum-wire band gaps, determined spectroscopically, are compared to the results of various theoretical analyses, and to previous experimental values for InP quantum dots and wires. The emergence of anisotropic colloidal quantum nanostructures, such as rods, wires, and tetrapods, have prompted current interest in the shape dependence of quantum confinement; that is, in how quantum confinement scales with the geometric dimensionality of confinement. However, systematic physical studies of quantum confinement in anisotropic nanostructures are limited, because specimens having narrow size distributions over a range of appropriate sizes have become available only recently. ${ }^{3-18}$ Thus, the general lack of quantitative experimental comparisons of corresponding sets of quantum wells, wires, rods, and dots has been due to a lack of appropriate high-quality specimens.

Because of such synthetic limitations, little is currently known experimentally about confinement effects in colloidal quantum wires. ${ }^{14,15,19}$ To our knowledge, the possible influences of synthetic method and surface ligation on the electronic structures of quantum wires have not been investigated.

Furthermore, only a few quantitative estimations of PL efficiencies in quantum wires have been 
reported. ${ }^{20-22}$ We have recently advanced a rudimentary physical model suggesting how the confinement effects in dots, wells, and wires should compare, ${ }^{14}$ but this model has not been thoroughly experimentally tested.

The work described herein provides a useful characterization of two-dimensional (2D) quantum confinement in semiconductor quantum wires. Our results establish that PL efficiencies in colloidal quantum wires are inherently low, and that the surface passivation can influence quantum-wire band gaps to a small but experimentally significant extent.

\section{Experimental section}

Materials. The precursor tris(trimethylsilyl)phosphine $\left(\mathrm{P}\left(\mathrm{SiMe}_{3}\right)_{3}\right)$ was prepared by a literature method. ${ }^{23}$ Stock solutions of $\mathrm{P}\left(\mathrm{SiMe}_{3}\right)_{3}$ (containing $0.40-0.42 \mathrm{mmol} \mathrm{P}\left(\mathrm{SiMe}_{3}\right)_{3} / \mathrm{mL}$ polydecene) were prepared and stored in a refrigerator for later use. Stock solutions of Bi catalyst nanoparticles (containing $0.04 \mathrm{mmol} \mathrm{Bi}$ atoms/g solution) were prepared as described previously; ${ }^{15}$ the detailed synthesis of a variety of sizes will be reported elsewhere. Tri-n-octylphosphine (TOP, 90\%), di- $n$ octylamine (DOA, 98\%), 1-hexadecylamine (HDA, 90\%), tri-n-octylphosphine oxide (TOPO, 90\%), and hydrogenated poly(1-decene) were purchased from Aldrich. HDA and TOPO were vacuum distilled at $200^{\circ} \mathrm{C}$; the other reagents were used as received. 1-Octylphosphonic acid (OPA, 99\%) was purchased from Lancaster, and used as received. Preparation of indium myristate $\left(\operatorname{In}(\operatorname{myr})_{3}\right)$ and a stock solution of it are described in the Supporting Information, as are preparations of crystalline $\mathrm{InPO}_{4} \cdot 2 \mathrm{H}_{2} \mathrm{O}$ and amorphous $\mathrm{InPO}_{4}$ hydrate.

Synthesis of InP quantum wires. All synthetic steps were conducted under dry, $\mathrm{O}_{2}$-free $\mathrm{N}_{2}(\mathrm{~g})$, but the isolation and purification steps were conducted in the ambient atmosphere. The quantities of reagents used are recorded in Table 1 (Supporting Information). In a typical preparation, the $\operatorname{In}(\mathrm{myr})_{3}$ 
stock solution, HDA, OPA, TOP, TOPO (in some experiments), and polydecene ( $3 \mathrm{~g}$ ) were loaded into a Schlenk reaction tube. In a separate vial, the Bi nanoparticle solution and DOA (in some experiments) were diluted with polydecene to $0.5 \mathrm{~mL}$, and the vial was septum capped. The $\mathrm{P}\left(\mathrm{SiMe}_{3}\right)_{3}$ stock solution $(0.5 \mathrm{~mL})$ and the Bi-nanoparticle-polydecene mixture were loaded into the same 3-mL syringe. The reaction mixture in the Schlenk tube was degassed under vacuum $\left(10^{-3}\right.$ torr $)$ at $\sim 100{ }^{\circ} \mathrm{C}$ for $5 \mathrm{~min}$, and then inserted into a preheated salt bath $\left(\mathrm{NaNO}_{3} / \mathrm{KNO}_{3}, 46: 54\right.$ by weight), held at a desired temperature (240-330 ${ }^{\circ} \mathrm{C}$; see Table 1). The tube was allowed to equilibrate for 1-2 min, and then the $\mathrm{P}\left(\mathrm{SiMe}_{3}\right)_{3}$ - $\mathrm{Bi}$ nanoparticle mixture was quickly injected into the tube. The color of the reaction mixture immediately turned red and finally to reddish brown (3-4 min). The reaction tube was withdrawn from the bath at 10 or 15 min after the injection and allowed to cool to room temperature. Black precipitates were observed in the mixtures, which were shown to be the InP quantum wires.

The black InP quantum-wire precipitate was isolated from the partially solidified reaction mixture by adding toluene (ca. $5 \mathrm{~mL}$ ) and isopropanol (ca. $5 \mathrm{~mL}$ ) to the mixture, followed by centrifugation of the mixture and decanting of the supernatant. The quantum wires were purified by redispersion in a mixture of toluene (ca. $2 \mathrm{~mL})$ and isopropanol $(\mathrm{ca} .4 \mathrm{~mL})$ upon sonication in a cleaning bath, followed by centrifugation and decanting the supernatant. One such redispersion-centrifugation purification cycle was generally sufficient to prepare quantum-wire specimens for absorption spectroscopy. Two redispersion-centrifugation purification cycles produced cleaner specimens for TEM analyses. After purification the InP quantum wires could be redispersed in pure toluene to form uniform and optically clear light-brown solutions for spectroscopic and TEM analyses. After a few hours the quantum wires began to reprecipitate from the toluene dispersions; however, they were easily redispersed by gentle shaking. The UV-visible spectra showed no changes after such samples were allowed to stand for two weeks in capped cuvettes. 
The percent yield of wires was estimated from a representative synthesis employing $0.21 \mathrm{mmol}$ of $\mathrm{P}\left(\mathrm{SiMe}_{3}\right)_{3}$ as the limiting reagent and 10.2-nm Bi nanoparticles, which produced 6-nm-diameter wires. The wires were isolated and purified as described above, and dried under vacuum $(1 \mathrm{~h})$ to yield $12 \mathrm{mg}$. The amount of $\mathrm{InP}$ in the isolated solid was calculated to be $8 \mathrm{mg}(0.06 \mathrm{mmol})$ by assuming that capping ligands were attached only to surface In atoms, that every surface In atom was bound to one ligand, equal numbers of surface In and P atoms, and the measured ratio of surface ligands (see Recovery and characterization of ligands on the surface of InP quantum wires in the Supporting Information). Consequently the yield was estimated at $30 \%$. Thicker wires ( $\sim 10-$ nm diameters) usually gave higher yields, whereas thinner wires ( $\sim 5$-nm diameters) gave lower yields.

Photochemical etching of InP quantum wires with HF - general conditions. The etching experiments were performed in air in 30-mL transparent NALGENE ${ }^{\circledR}$ Teflon FEP bottles using a modified literature method. ${ }^{1}$ The HF etching solution $(2.40 \mathrm{M})$ was prepared by diluting $1.065 \mathrm{~g}$ of HF (50 wt. \%) in a mixture of $n$-butanol $(10 \mathrm{~mL})$ and water $(140 \mathrm{mg})$. The InP wires employed were typically $6 \mathrm{~nm}$ in diameter, and constituted the entire batch from a single preparation in an estimated quantity of $0.06 \mathrm{mmol}$ (see above).

Thinning of InP wires by photochemical etching. Thinning of InP quantum wires by HF etching was performed under room light or with a 100-W tungsten lamp. The lamp was equipped with a long-pass filter having a cutoff wavelength of $700 \mathrm{~nm}$. The wires were dispersed in toluene (1-3 mL) and combined with $n$-butanol (6-9 mL), isooctane ( $3 \mathrm{~mL})$, and TOPO (200-500 mg, 0.5-1.3 mmol). The HF stock solution (0.4-1.2 mL) was then added with stirring, and the mixture was exposed to room light (4-12 h) or the filtered tungsten lamp (6-68 h). In some cases, additional aliquots of reagents were added at later times after spectroscopic monitoring. The precise quantities of reagents and illumination periods used in each experiment are recorded in Table 2 (Supporting Information). The thinned wires 
were isolated from the reaction mixture by addition of $\mathrm{CH}_{3} \mathrm{CN}(5 \mathrm{~mL})$, followed by centrifugation and decanting of the supernatant. The isolated wires were redispersed in toluene for subsequent spectroscopic and TEM analyses.

\section{Photo-oxidation of InP wires by photochemical etching. Photo-oxidation of InP wires} occurred by HF etching under a 300-W xenon lamp. The lamp was equipped with a long-pass filter having a cutoff wavelength of $700 \mathrm{~nm}$, as above. InP wires $(0.06 \mathrm{mmol})$ were dispersed in a mixture of $n$-butanol $(9 \mathrm{~mL})$, isooctane $(3 \mathrm{~mL})$, toluene $(3 \mathrm{~mL})$, and TOPO (300-500 $\mathrm{mg}, 0.8-1.3 \mathrm{mmol})$. The HF stock solution $(0.5-1.2 \mathrm{~mL})$ was then added with stirring, and the mixture was exposed to the filtered xenon lamp $(6-210 \mathrm{~h})$. The precise quantities of reagents and illumination periods used in each experiment are recorded in Figure 5c. Aliquots $(1-2 \mathrm{~mL})$ of the reaction solution were removed at various time intervals for spectroscopic monitoring. The photo-oxidized wires were isolated from the reaction mixture by addition of $\mathrm{CH}_{3} \mathrm{CN}(1 \mathrm{~mL})$, followed by centrifugation and decanting of the supernatant. The isolated wires were redispersed in toluene for subsequent spectroscopic and TEM analyses.

As control experiments, etching under room light or in darkness was carried out similarly. InP wires were also etched by tetrabutylammonium fluoride analogously to the above procedure (see Figure S21, Supporting Information).

In a separate set of aging experiments (Figure 8), InP quantum wires were HF-photo-oxidized under the filtered xenon lamp (as described above), isolated and purified, redispersed in toluene under air in borosilicate tubes, and then exposed to room light. Spectroscopic measurements were made every several days. 


\section{Results}

Synthesis. Our previous synthesis of InP quantum wires used a non-commercially available single-source precursor and a large amount of polymer stabilizer, ${ }^{14}$ both of which generated inconveniences. Here we report a simpler SLS synthesis, affording InP quantum wires passivated by traditional quantum-dot surfactants (eq 1; see the Experimental Section for abbreviation definitions). The experimental conditions, such as the precursor ratio and the amounts and identities of the various surfactants employed, required careful optimization for production of the best-quality quantum wires, as discussed below (see Table 1, Supporting Information).

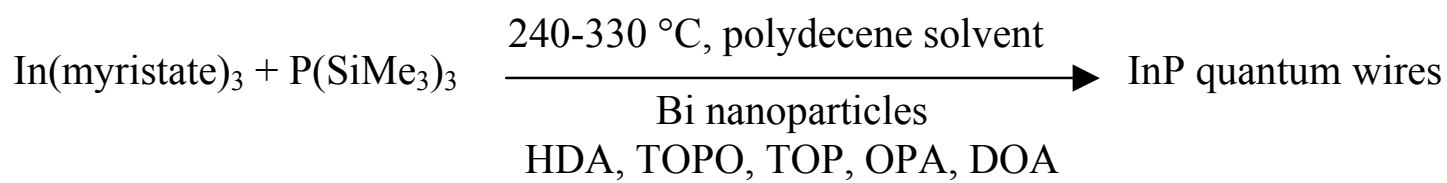

The $\mathrm{In}(\mathrm{myr})_{3} / \mathrm{P}\left(\mathrm{SiMe}_{3}\right)_{3}$ ratio determined the phase purity of the InP wires. When the indium precursor was used in large excess $(\mathrm{In} / \mathrm{P}$ ratio $=1.6-2.4)$, the wires were contaminated with crystalline (cubic) $\mathrm{In}_{2} \mathrm{O}_{3}$ as a major impurity component. The formation of $\operatorname{In}_{2} \mathrm{O}_{3}$ may have been due in part to adventitious water, as its quantity was reduced by the use of distilled TOPO and HDA. However, this oxide component was eliminated by using In/P precursor ratios of less than 1.4 . The optimal In/P precursor ratio was found to be in the range of 1.1-1.3.

The surfactants HDA and OPA were essential to the production of InP wires with high yield and high quality. HDA enhanced the crystallinity of the wires, and promoted the formation of narrow diameter distributions. Syntheses performed in the presence of HDA but the absence of OPA produced only short InP rods by SLS growth, in mixtures with InP rod clusters that presumably resulted from homogeneous nucleation. Addition of the strongly binding surfactant $\mathrm{OPA}^{7}$ quenched the growth of those homogeneous nuclei, thereby precluding formation of the rod clusters and allowing the 
competitive SLS growth of longer InP quantum wires. The optimal In(myr) 3 /OPA ratio was found to be 1.5. The use of larger amounts of OPA decreased the quality of the InP wires formed, and the selectivity for wire formation in relation to other product morphologies.

The surfactants TOPO and TOP were unnecessary to the growth of high-quality InP quantum wires having large diameters of $\geq 10 \mathrm{~nm}$. However, such wires grown in the absence of TOPO and TOP (using HDA and OPA only) were smaller in diameter than those grown from identical Bi nanoparticles in the presence of all four surfactants. For example, InP quantum wires grown from 44-nm Bi catalyst particles exhibited a mean diameter of $15 \mathrm{~nm}$ in the absence of TOPO and TOP, and $19 \mathrm{~nm}$ in the presence of all four surfactants. The growth of good-quality quantum wires having diameters of $<10$ $\mathrm{nm}$ required the use of all four surfactants.

The surfactant DOA was commonly added to the Bi nanoparticles to reduce their agglomeration tendencies at high reaction temperatures. Consequently, the use of DOA resulted in generally narrower quantum-wire diameter distributions, particularly in syntheses employing smaller-diameter Bi nanoparticles. The optimal surfactant ratio was found to be HDA/TOPO/myristate/TOP/OPA/DOA = 16.7/4.6/6.0/1.8/1.2/1.0.

The syntheses were conducted in the range of $240-330{ }^{\circ} \mathrm{C}$, with temperatures at the high end necessary for the growth of large-diameter wires, and temperatures at the low end for small-diameter wires. Higher growth temperatures generally improved the crystallinity of the quantum wires at the expense of broader diameter distributions. For smaller-diameter wires, high growth temperatures were also detrimental to morphological control.

The amount of Bi-nanoparticle catalyst employed also influenced the quality of the smallerdiameter quantum wires. Too little catalyst resulted in lower selectivity for the wire morphology. Too much catalyst resulted in short wires. The optimal $\mathrm{P}\left(\mathrm{SiMe}_{3}\right)_{3} / \mathrm{Bi}$ ratio was found to be $100-150$. Larger 
amounts of Bi were employed in the growth of larger-diameter wires to account for the smaller number of Bi nanoparticles per mmol Bi in the large-nanoparticle stock solution $(d=44 \mathrm{~nm})$.

The InP quantum wires were characterized by TEM, selected-area electron diffraction (SAED), XRD (see Fig. S12, Supporting Information), and EDX (Fig. S19). The wires possessed mean diameters ranging from 4.3-19.0 nm; TEM images of representative examples across this range are shown in Figure 1. The diameters were controlled primarily by the size of the Bi-nanoparticle catalysts employed; however, as noted above the surfactant selection was also influential. Thinner wires $(\mathrm{d}<5 \mathrm{~nm})$ exhibited lengths of 50-500 nm, and typically were kinked. Thicker wires exhibited lengths of up to 10 $\mu \mathrm{m}$, and were much straighter. Analysis of SAED patterns established that the wire long axes were oriented along the [111] direction of the zinc-blende crystal structure. Apart from kinks and stacking faults, which were observed to be prevalent in the thinner and thicker wires, respectively, the quantum wires exhibited near single-crystal character.

As described previously, the best results were obtained by using a surfactant mixture that included HDA, TOPO, TOP, and OPA. An analysis was undertaken to determine the actual surface coverage of the quantum wires after growth. A purified quantum-wire specimen was digested in acid, and the liberated surfactant molecules were extracted for mass-spectrometric analysis. The $\mathrm{HDA} / \mathrm{TOPO} /$ myr surfactant ratio in the recovered mixture was found to be $15 / 1 / 4$, which compares fairly closely to the ratio of $14 / 4 / 5$ employed in the synthetic precursors. In this analysis, the surfactants TOP and OPA were not found. Thus, HDA was the predominant surfactant stabilizing the wires. Although OPA is a strongly binding surfactant, ${ }^{7}$ it was used in small amounts relative to HDA, TOPO, and myristate, and was apparently displaced by the other surfactants.

The absorption and emission properties of the wires, and their correlations with the band gaps, passivation, and structural features of the wires are discussed in subsequent sections. 
Photochemical Thinning of InP Quantum Wires. When synthesized as described above, the InP quantum wires gave no detectable room-temperature PL. To improve the PL of the wires, photochemical etching in the presence of HF (and air) was undertaken under the general conditions previously reported by Talapin and coworkers. ${ }^{1,24}$ Our initial efforts employed irradiation with lowerintensity light sources, which were either a filtered tungsten lamp $(100 \mathrm{~W}, \lambda \geq 700 \mathrm{~nm})$, or unfiltered room fluorescent lighting. Under these comparatively mild conditions (Table 2, Supporting Information), a thinning of the InP quantum wires was achieved without detectable enhancements in their PL. We note that related decreases in the diameters of InP quantum dots photochemically etched under similar conditions were previously reported, along with significant enhancements in the PL quantum yields. ${ }^{1,24}$

TEM images of the wires (Fig. S15) confirmed that thinning had occurred without other gross structural changes; they remained near-single-crystalline InP quantum wires. Furthermore, the absorption spectra of the wires retained resolvable excitonic features (see below, and Fig. S16). The initial diameters of the wires were in the range of 5.6-6.5 nm. Upon photochemical thinning, the diameters decreased to the range of 4.5-6.2 $\mathrm{nm}$. Consequently, the photochemical-thinning experiments provided additional specimens for the systematic study of the diameter-dependent absorption spectra of the quantum wires.

Absorption Spectroscopy and Diameter Dependence of the Quantum-Wire Band Gap. The absorption spectra of InP quantum wires prepared above having diameters in the range of 4.3-8.8 nm are shown in Fig. 2a. The lowest-energy excitonic features were extracted by background fitting and subtraction as previously described (see also the Supporting Information). ${ }^{14}$ Each backgroundsubtracted peak (Fig. 2b) was fit with a Guassian function, and the center of the fit (ranging from $\lambda=$ $747-835 \mathrm{~nm}$ ) was taken to be the band gap of the wire specimen (Table 1, Supporting Information). The 
excitonic features (band gaps) shifted to lower energy with increasing wire diameter, as expected and as observed previously in InP quantum wires ${ }^{14,19}$ and dots. ${ }^{2,25,26}$ Spectra collected from larger diameter wires contained no resolvable excitonic features.

Band-gap data for the as-synthesized InP quantum wires passivated with traditional quantumdots surfactants (HDA, TOPO, and TOP), photochemically thinned InP wires, previously synthesized polymer-passivated InP wires, and InP quantum dots are compared in Fig. 3. The data are plotted as $\Delta E_{g}$, the increase in the band gap above the bulk value, vs. $d^{-2}$, the inverse-square of the dot or wire diameter, in accord with a previously proposed model. ${ }^{14}$ In this model, the size dependences of the band gaps for dots and wires are estimated by calculating the kinetic confinement energies of electron-hole pairs using simple particle-in-a-box expressions for infinitely long cylinders, and spheres. ${ }^{14}$ The model predicts that the $\Delta E_{g^{-} \text {-vs. }}-d^{-2}$ plots should be linear, with $A_{\text {wire }}: A_{\text {dot }}$ slope ratios of $0.585 .{ }^{14}$

The dot and wire data in Fig. 3a are well fit linearly, as previously reported. ${ }^{14}$ The three sets of wire data plot very closely together. Only a small offset is evident between the band gaps determined for the polymer- and surfactant-passivated wires, which presumably reflects a small difference in the confinement potential imparted by the differing surface passivation in the two cases. The slope ratios calculated are $A_{\text {wire }}: A_{\mathrm{dot}}=0.66 \pm 0.03$ for the surfactant-passivated wires, and $A_{\text {wire }}: A_{\mathrm{dot}}=0.62 \pm 0.03$ for the polymer-passivated wires, both of which are close to the theoretical prediction of $A_{\text {wire }}: A_{\text {dot }}=0.585$. A slope was not determined from the data for the HF-thinned wires (green squares in Fig. 3) because the diameter range was insufficient to allow reliable fitting.

The theoretical model referred to above is quite rudimentary, and assumes the effective-mass approximation, including parabolic band shapes and bulk (size-independent) effective masses, and infinite potential barriers at the dot or wire surfaces. Furthermore, this effective-mass-approximation, particle-in-box (EMA-PIB) model ignores the Coulombic attraction between excitonic electron-hole 
partners. Although the experimental $\Delta E_{g}$-vs.- $d^{-2}$ slope ratios are close to the predicted value, the absolute dot and wire slopes observed experimentally and predicted by the model (not shown) are far from agreement, as EMA-PIB models are known to overestimate quantum-confined band gaps. ${ }^{27,28}$ Additionally, the experimental Fig.3a lines do not extrapolate through the origin as they should, consistent with the disappearance of quantum-confinement at large dot or wire diameters.

Consequently, the InP quantum-wire band gaps have been investigated at higher levels of theory. We previously reported calculation of InP quantum-wire band gaps by the semiempirical psuedopotential method (SEPM). ${ }^{14}$ By that approach, band-gaps were calculated for several specific diameters in the range of $2.5-8.6 \mathrm{~nm}$, and the results were fit with the expression $\Delta E_{g}=A d^{-n}$, yielding values of $A_{\mathrm{SEPM}}=2.49 \pm 0.09 \mathrm{eV} \mathrm{nm}^{1.45}$ and $n_{\mathrm{SEPM}}=1.45 \pm 0.03$. This fitted curve is plotted vs. $d^{-2}$ in Fig. 3b.

Subsequently, Wang and Li reported the determination of quantum-dot and quantum-wire band gaps using density functional theory under the local-density approximation by implementing a chargepatching method (DFT-LDA-CPM), ${ }^{29}$ which affords ab-initio results at slightly greater computational cost than the SEPM. For the present work, band-gaps were calculated for several specific InP quantumwire diameters in the range of 2.3-8.2 nm using the DFT-LDA-CPM. As above, these results were fit with the expression $\Delta E_{g}=A d^{-n}$, yielding values of $A_{\mathrm{DFT}}=2.37 \pm 0.06 \mathrm{eV} \mathrm{nm}^{1.34}$ and $n_{\mathrm{DFT}}=1.34 \pm 0.02$. These $A_{\mathrm{DFT}}$ and $n_{\mathrm{DFT}}$ values differ slightly from those previously reported for InP quantum wires, which were fitted from band gaps calculated for smaller diameters in the range of 1.1-4.2 nm, ${ }^{29}$ which does not overlap with the experimental results described above. The fitted curve for the $A_{\mathrm{DFT}}$ and $n_{\mathrm{DFT}}$ values determined here is also plotted vs. $d^{-2}$ in Fig. $3 b$.

Several observations regarding the theoretical and experimental results in Fig. $3 \mathrm{~b}$ are intuitively attractive. First, at higher levels of theory $\Delta E_{g}$ does not scale precisely with $d^{-2}$ as indicated 
by the simple EMA-PIB model. Consequently, the $\Delta E_{g}$-vs.- $-d^{-2}$ plots are gentle curves that do intercept the origin, as they must. The difference between the two theoretical results represents the typical predictive power of the theory, and it also embodies the slightly different treatments of the nanowire surface in these two theoretical models. The SEPM and DFT-LDA-CPM as implemented above for quantum wires do not account for the electron-hole Coulomb attraction, and thus should overestimate the band gaps at smaller diameters as is evident. Even so, the experimental data fall largely on or between the two theoretical curves, constituting excellent agreement between experiment and the best available theoretical calculations for InP quantum wires. The experimentally measured band gaps are reasonable.

Photochemical Oxidation of InP Quantum Wires. As noted above, InP quantum wires $(d \approx 6$ $\mathrm{nm}$ ) were photochemically etched in the presence of HF (and air) with the goal of producing detectable PL from the wires. ${ }^{1,24}$ Our initial efforts produced only photothinning of the wires without enhancement of the PL. Photochemical etching with more-intense irradiation (300-W xenon lamp, $\lambda \geq 700 \mathrm{~nm}$ ) did elicit PL from the wire samples. However, our studies established that the InP wires were photooxidized under the conditions employed, affording amorphous indium phosphate wires with embedded dot- and rod-like domains of crystalline (residual) InP. The emission observed was produced by the embedded InP dots and rods. These conclusions are supported by the results below.

Monitoring of the absorption spectra of InP quantum wires during photochemical etching with the more-intense light source indicated a gradual blue shift of the first excitonic feature (Fig. 4a), consistent with wire thinning (see above). The feature eventually broadened and was no longer resolvable. During this process a peak emerged in the PL spectrum of the etched samples. Figure $4 \mathrm{~b}$ compares the absorption and emission spectra of a photochemically etched sample prior to the disappearance of the first excitonic feature in the absorption spectrum. Interestingly, the emission 
feature is blue-shifted from the absorption feature in an apparent "inverse" Stokes shift, which is nonphysical. The results established that the primary absorbing and emitting entities were not the same.

Figure 5 shows that the PL from the wire samples increased with increasing photochemicaletching time. Furthermore, the first emission feature that emerged near $1.7 \mathrm{eV}$ was progressively blue shifted. At later times, a second emission feature emerged near $1.9 \mathrm{eV}$, which was also progressively blue shifted. Significantly, these features did not appear in the region expected for emission from InP quantum wires having the expected diameters of 4.5-6.5 $\mathrm{nm}(<1.6 \mathrm{eV})$, but rather were consistent with emission from quantum rods $(1.7 \mathrm{eV})$ and dots $(1.9 \mathrm{eV})$ having approximate diameters of 4 and $3 \mathrm{~nm}$, respectively (see Table 3, Supporting Information). The results suggested a process that, while thinning the wires, was also transforming them into progressively smaller rod and then dot domains, which were responsible for the PL observed.

Insight into the nature of this transformation was gained from TEM images of the photochemically etched wires (Fig. 6). The wiry morphology of the nanostructures remained intact, and in many regions they retained long, wire-like segments of crystalline $\mathrm{InP}$ (see the dark wire in the middle of Fig. 6b). However, in other regions the wiry structures contained dark rod- and dot-sized nanocrystals of crystalline InP embedded in an amorphous matrix. The crystalline (dark) and amorphous (light) regions of the nanostructures were distinguished by electron diffraction in the TEM. (The amorphous regions were susceptible to electron-beam damage.) Consequently, the photochemical etching converted large segments of the InP quantum wires into amorphous domains containing small residual islands of crystalline InP.

The effective diameters of the wires were monitored by TEM as a function of etching time under the most-aggressive photochemical-etching conditions. While an initial blue shift in the absorption edge suggested initial wire thinning (see above and Fig. 4a), the TEM results established that the wire 
diameters actually increased with etching time (Fig. 7). These increases occurred concomitantly with the process generating the amorphous wire domains, suggesting a partial chemical conversion of the wires affording a new amorphous phase or phases. We will argue below that this chemical reaction is a photo-oxidation of InP to a phosphate or phosphates.

As noted above, the effective PL from the wires increased with etching time, as the emitting rod and dot domains were generated (Fig. 5a, c, d). In a separate series of experiments, InP quantum wires were photochemically etched for fixed periods of $14-48 \mathrm{~h}$, and then isolated from the etching mixture. The wires were then redispersed in toluene and allowed to stand under room light (and air). As shown in Fig. 8, the PL from these isolated wires continued to increase with time, but without additional blue shifting. The results suggested that upon removal of HF the sizes of the emitting domains were no longer changing, but that the interface between the amorphous matrix and the emitting domains was rearranging in some manner that reduced the numbers of trap sites contributing to the nonradiative relaxation of photogenerated carriers.

We next sought to identify the amorphous matrix generated by the photochemical-etching process. Figure 9 contains In $3 d$ and P $2 p$ core-level x-ray photoelectron spectra (XPS) collected from the photochemically etched wires. The data were obtained with a sampling depth of ca. $5 \mathrm{~nm}$, which is a significant fraction of the mean wire diameter. Both the In $3 d$ and P $2 p$ spectra were well fit as a mixture of InP and indium phosphate, $\mathrm{InPO}_{4}$, or metaphosphate, $\operatorname{In}\left(\mathrm{PO}_{3}\right)_{3} .{ }^{30}$ The In $3 d$ and $\mathrm{P} 2 p$ binding energies reported for $\mathrm{InPO}_{4}$ and $\mathrm{In}\left(\mathrm{PO}_{3}\right)_{3}$ are very close, such that they cannot be distinguished by the peak position. However, the XPS data give a ratio between oxidized indium and phosphorus of almost unity, suggesting that $\mathrm{InPO}_{4}$ is predominant (see Table 5, Supporting Information). The amount of oxygen is also in good agreement with $\mathrm{InPO}_{4}$. The etching mixtures contained TOPO, so the contribution of TOPO to the XPS peaks assigned to $\mathrm{InPO}_{4}$ or $\operatorname{In}\left(\mathrm{PO}_{3}\right)_{3}$ must be considered. However, an 
analysis of the surface ligands after photochemical etching indicated only small residual amounts of TOPO (see the Supporting Information). Consequently, the amorphous matrix may be either $\mathrm{InPO}_{4}$, or a mixture of $\mathrm{InPO}_{4}$ with a small amount of $\mathrm{In}\left(\mathrm{PO}_{3}\right)_{3}$, resulting from the partial photochemical oxidation of the InP wires. The results are consistent with a prior report by Talapin et al., ${ }^{24}$ which found that photochemical etching of InP quantum dots in the presence of HF generated phosphorus-oxide species on the dot surfaces.

Analysis by infrared (IR) spectroscopy was undertaken to provide further support for the above assignment. Figure 10 contains IR spectra for InP nanowires before and after HF photo-oxidation. These spectra were compared to those of the ligands potentially present, myristate, HDA, and TOPO, and to those of the independently obtained candidate phases $\mathrm{InF}_{3}$ (indium trifluoride), crystalline $\mathrm{InPO}_{4} \cdot 2 \mathrm{H}_{2} \mathrm{O}$, and amorphous $\mathrm{InPO}_{4}$. A reasonably strong correlation was evident between the spectra for the photo-oxidized nanowires and the independently prepared phosphate phases. The phosphate group has a characteristic broad absorption in the range of $940-1120 \mathrm{~cm}^{-1} .31$ A corresponding feature in the spectrum of the photo-oxidized wires extends over the broader range of $900-1300 \mathrm{~cm}^{-1}$. However, the metaphosphate $\operatorname{In}\left(\mathrm{PO}_{3}\right)_{3}$ exhibits a broad absorption over the range of $1240-1320 \mathrm{~cm}^{-1}$, and a lowerenergy feature at $640-790 \mathrm{~cm}^{-1} .^{32}$ Consequently, the IR spectrum of the photo-oxidized wires is consistent with a mixture of indium phosphate and metaphosphate phases, with some contribution by capping ligands.

\section{Discussion}

We have been conducting systematic studies of the diameter dependence of the band gap in semiconductor quantum wires for comparison to the related dependences in corresponding sets of quantum dots, rods, and wells. To date we have published two such studies, which investigated the 
quantum-confinement effects in $\mathrm{InP}^{14}$ and $\mathrm{CdSe}^{15}$ quantum wires. We also explicitly acknowledge the related studies of InP quantum wires having larger diameters $(d=10-50 \mathrm{~nm})$ by Lieber and coworkers, ${ }^{19}$ the results of which are complementary to and consistent with those reported here. The goal of our work is to establish experimentally how the shape of a colloidal quantum nanostructure influences quantum confinement and its size dependence.

In drawing comparisons between corresponding sets of dots, rods, wires, and wells, the confinement potential is implicitly assumed to depend primarily on the size and shape of the nanostructure, and not significantly upon the surface ligands and passivation. Studies of variously passivated quantum dots have demonstrated only a small effect of surface ligation on the band gap. ${ }^{33,34}$ However, the influence of surface ligation on quantum confinement in quantum wires has not to our knowledge been previously investigated. In this work a small but significant effect of surface ligation on the confinement potential was observed.

Figure 3a compares the band gaps of variously passivated InP quantum wires. The pink data and line correspond to InP quantum wires passivated primarily with HDA, whereas the blue data and line correspond to wires passivated by the polymer poly(1-hexadecene ${ }_{0.67}$-co-vinylpyrrolidinone - $\left._{0.33}\right)$. The confinement energies ( $\Delta E_{g}$ values) differ by $20-40 \mathrm{meV}\left(10-16 \%\right.$ of $\left.\Delta E_{g}\right)$ between these two sets of wires, depending on diameter. These differences are larger than the estimated error of $10 \mathrm{meV}$ in the measurement of $\Delta E_{g}$, and are therefore small but significant.

The precise origin of the small surface-ligation effect on $\Delta E_{g}$ is unknown, but presumably reflects delocalization of electron and/or hole wave functions, to varying degrees, outside the semiconductor core of the wires and into the ligand shell or environment. ${ }^{35}$ Consequently, the assumption of an infinite potential barrier at the semiconductor-nanostructure surface often employed in theoretical calculations is not completely valid, although a reasonable approximation. Figure $3 b$ shows 
that our experimental $\Delta E_{g}$ values compare closely to the predictions of SEPM and DFT-LDA-CPM calculations, suggesting that we have successfully extracted the positions of the first excitonic features, and thus properly measured the band gaps, in the InP quantum wires.

In corresponding sets of equally passivated semiconductor quantum dots, rods, and wires, the PL efficiencies should be highest in the dots, and lowest in the wires. Quantum-dot surface passivation has now advanced to routinely afford specimens having PL quantum yields of $\geq 60 \%$. By comparison, the PL quantum yields in quantum rods, typically ranging from $15-30 \%,{ }^{9,12}$ are respectable but lower than those in dots. Banin and coworkers have shown that PL efficiencies in quantum rods decrease with increasing rod length, and have argued that relaxation of the third confinement dimension in rods allows the electron and hole wave functions to relax apart, leading to a lower probability of radiative recombination. ${ }^{11,13}$ In quantum wires, the third dimension of confinement vanishes completely, allowing photogenerated electrons and holes to diffuse to some extent along the wire length, increasing the probability they will encounter surface-trapping defects and recombine nonradiatively. Consequently, PL efficiencies should be lowest in semiconductor quantum wires, and measurements to date have indicated ensemble quantum yields in wires of only $0.1-2 \% .{ }^{20-22}$

As described above, the InP quantum wires as-synthesized gave no detectable room-temperature PL. We attempted to enhance the PL using the HF-etching method first described by Mićić, Nozik, and coworkers. ${ }^{2}$ This method was further developed and implemented photochemically by Talapin and coworkers, which was shown to very effectively enhance the PL of InP quantum dots from initial quantum yields of $0.1-0.3 \%$ to $20-40 \% .{ }^{1}$ Irradiation intensities lower than those used by Talapin induced only photochemical thinning of the wires, without enhancement of PL. Irradiation intensities similar to those used by Talapin induced a photo-oxidation that degraded the wire nanostructures to 
leave residual, embedded, luminescent, rod and dot domains. Consequently, quantum-wire luminescence was not achieved.

These findings identify an interesting contrast in surface-etching procedures for quantum dots and wires. Surface etching of dots may lead to decreases in dot diameters, but will not affect the confinement dimensionality. Quantum dots are 3D confined before and after surface-etching procedures that enhance PL. In contrast, etching procedures conducted on quantum wires may increase the confinement dimensionality, from 2D to anisotropic and then roughly isotropic 3D confinement.

Our results differ from those described by van Vugt and coworkers, ${ }^{22}$ who previously studied HF photoetching of InP nanowires having large diameters $(d=30-60 \mathrm{~nm})$, far outside the quantumconfinement regime. The PL quantum yields of these large-diameter wires were reported to be 0.01 $1.0 \%$ in their initial, as-synthesized state. Irradiation in the presence of HF produced PL enhancements of on the order of 10-fold. Interestingly, the wires exhibited blue-shifted luminescence both before and after photochemical surface re-passivation, with greater blue shifts after. These blue shifts were attributed to exciton interactions with surface charges or dipoles. As the magnitudes of the blue shifts were comparatively small, they are unlikely due to photochemical generation of dot and rod domains, as we observed. Thus, larger-diameter nanowires, as expected, will be less susceptible to the dimensionality changes that can occur upon etching of small-diameter, quantum wires.

\section{Conclusion}

PL efficiencies in quantum wires are intrinsically lower than those in quantum dots and rods because of the reduced confinement dimensionality. Surface-etching strategies that raise PL efficiencies in dots will be more difficult to apply successfully to wires, as they can induce changes in confinement dimensionality by transforming wires into rods and dots. Consequently, increasing the low PL 
efficiencies so far achieved in wires remains one of the most significant challenges confronting the colloidal-quantum-wire field.

Acknowledgment. We thank Prof. Richard A. Loomis and Mr. John Glennon for helpful discussions, and Prof. Dewey Holten and Prof. Chris Kirmaier for training in PL spectroscopy. We are grateful to the National Science Foundation for funding this work under grant no. CHE-0518427, and to NASA for funding under grant no. NCC 2-1363.

Supporting Information Available: Additional methods and supporting results. This material is free of charge via the Internet at http://pubs.acs.org.

\section{References}

(1) Talapin, D.V.; Gaponik, N.; Borchert, H.; Rogach, A.L.; Haase, M.; Weller, H. J. Phys.Chem. B 2002, 106, 12659-12663.

(2) Mićić, O. I.; Sprague, J.; Lu Z.; Nozic, A. J. Appl. Phys. Lett. 1996, 68,3150-3152.

(3) Peng, X.; Manna, L.; Yang, W.; Wickham, J.; Scher, E.; Kadavanich, A.; Alivisatos, A. P. Nature 2000, 404, 59-61.

(4) Manna, L.; Scher, E. C.; Alivisatos, A. P. J. Am. Chem. Soc. 2000, 122, 12700-12706.

(5) Li, L.-S; Hu, J.; Yang, W.; Alivisatos, A. P. Nano Lett. 2001, 1, 349-351.

(6) Hu, J.; Li, L.-S; Yang, W.; Manna, L.; Wang, L.-W; Alivisatos, A. P. Science 2001, 292, 2060 2063.

(7) Peng, Z. A.; Peng, X. J. Am. Chem. Soc. 2001, 123, 1389-1395.

(8) Peng, Z. A.; Peng, X. J. Am. Chem. Soc. 2002, 124, 3343-3353.

(9) Manna, L.; Scher, E. C.; Li, L.-S; Alivisatos, A. P. J. Am. Chem. Soc. 2002, 124, 7136-7145. 
(10) Manna, L.; Milliron, D. J.; Meisel, A; Scher, E. C.; Alivisatos, A. P. Nature Mater. 2003, 2, 382385.

(11) Kan, S.; Mokari, T.; Rothenberg, E.; Banin, U. Nature Mater. 2003, 2, 155-158.

(12) Mokari, T.; Banin, U. Chem. Mater. 2003, 15, 3955-3960.

(13) Kan, S.; Aharoni, A.; Mokari, T.; Banin, U. Faraday Discuss. 2004, 125, 23-38.

(14) Yu, H.; Li, J.; Loomis, R. A.; Wang, L.-W; Buhro, W. E. Nature Mater. 2003, 2, 517-520.

(15) Yu, H.; Li, J.; Loomis, R. A.; Gibbons, P. C.; Wang, L.-W; Buhro, W. E. J. Am. Chem. Soc. 2003, $125,16168-16169$.

(16) Yu, H.; Buhro, W. E. Adv. Mater. 2003, 15, 416-419.

(17) Wang, F.; Dong, A.; Sun, J.; Tang, R.; Yu, H.; Buhro, W. E. Inorg. Chem. 2006, 45, 7511-7521.

(18) Shweky, I.; Aharoni, A.; Mokari, T.; Rothenberg, E.; Nadler, M.; Popov, I.; Banin, U. Mater. Sci. Eng. C 2006, 26, 788-794.

(19) Gudiksen, M. S.; Wang, J.; Lieber, C. M. J. Phys. Chem. B 2002, 106, 4036-4039.

(20) Protasenko, V. V.; Hull, K. L.; Kuno, M. Adv. Mater. 2005, 17, 2942-2949.

(21) Joo, Jin; Son, J. S.; Kwon, S. G.; Yu, J. H.; Hyeon, T. J. Am. Chem. Soc. 2006, 128, 5632-5633.

(22) van Vugt, L. K.; Veen, S. J.; Bakkers, E. P. A. M.; Roest, A. L.; Vanmaekelbergh, D. J. Am.

Chem. Soc. 2005, 127, 12357-12362.

(23) Askham, F.R.; Stanley, G.C; Marques, E.C. J. Am. Chem. Soc. 1985, 107, 7423-7431.

(24) Adam, S.; Talapin, D. V.; Borchert, H.; Lobo, A.; McGinley, C.; de Castro, A. R. B.; Haase, M.; Weller, H.; Möller, T. J. Chem. Phys. 2005, 123, 084706.

(25) Mićić, O. I.; Jones, K. M.; Cahill, A.; Nozic, A. J. J. Phys. Chem. B 1998, 102, 9791-9796.

(26) Mićić, O. I.; Ahrenkiel, S. P.; Nozik, A. J. Appl. Phys. Lett. 2001, 78, 4022-4024.

(27) Brus, L. E. J. Chem. Phys. 1984, 80, 4403-4409. 
(28) Yoffe, A. D. Advances in Physics 2002, 51, 799-890.

(29) Li, J.; Wang, L.-W. Phys. Rev. B 2005, 72, 125325.

(30) Hollinger, G.; Bergignat, E. J. Vac. Sci. Technol. A 1985, 3, 2082-2088.

(31) Nyquist, R. A.; Kagel, R. O. In The Handbook of Infrared and Raman Spectra of Inorganic Compounds and Organic Salts; Academic Press: San Diego, 1997; Vol. 4, Infrared Spectra of Inorganic Compounds.

(32) Llieva, D.; Kovacheva, D.; Petkov, C.; Bogachev, G. J. Raman Spectrosc. 2001, 32, 893-899.

(33) Kuno, M; Lee, J. K.; Dabbousi, B. O.; Mikulec, F. V.; Bawendi, M .G. J. Chem. Phys. 1997, 106, 9869-9882.

(34) Majetich, S. A.; Carter, A.C. J. Phys. Chem. 1993, 97, 8727-8731.

(35) Rabani, E.; Hetenyi, B.; Berne, B. J.; Brus, L .E. J. Chem. Phys. 1999, 110, 5355-5369. 


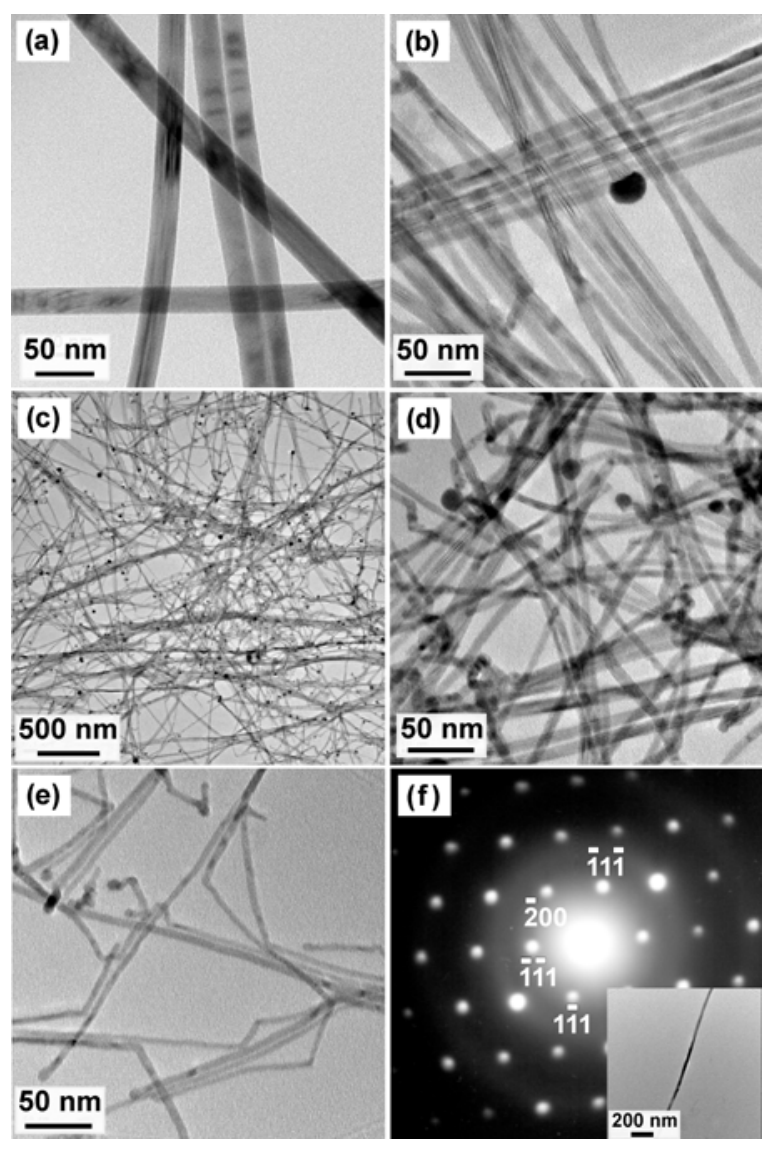

Figure 1. TEM images of InP wires (mean diameter \pm one standard deviation in the diameter distribution, expressed as a percentage of the mean diameter). (a) $19.0 \mathrm{~nm} \pm 24.2 \%$, (b and c) $11.2 \mathrm{~nm} \pm$ $17.5 \%$, (d) $6.9 \mathrm{~nm} \pm 16.1 \%$, (e) $5.6 \mathrm{~nm} \pm 13.2 \%$, and (f) electron-diffraction pattern in an [011] zone from the quantum wire shown. The observed reflections are indexed, demonstrating the [111] orientation of the wire. 


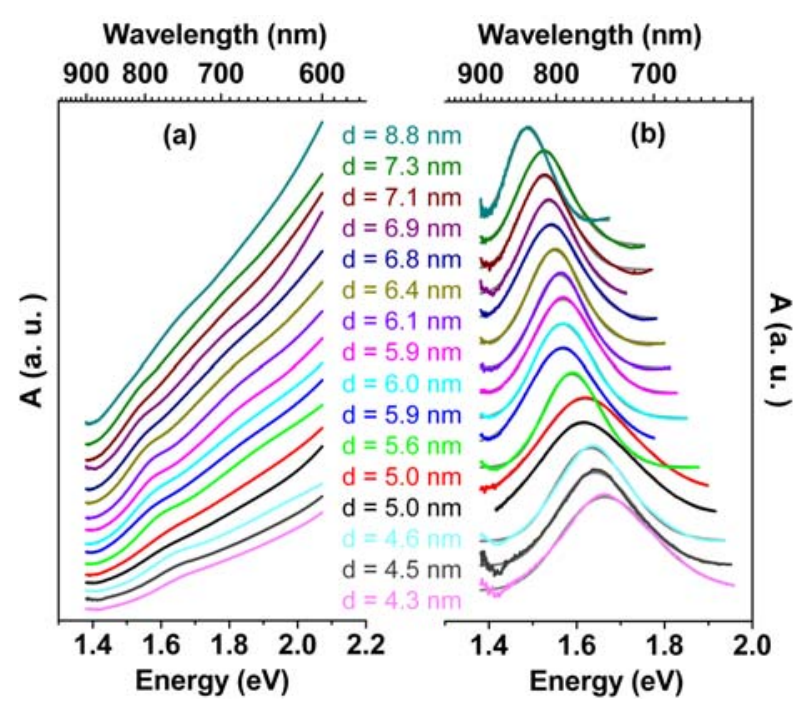

Figure 2. (a) Representative absorption spectra of InP wires; wire diameters ( $d$ values) are given in the key; and (b) Lowest-energy excitonic peaks extracted by nonlinear least-squares fitting and background subtraction (various colors), and the Gaussian fits to those peaks (gray). Wires synthesized under various conditions are included (see Table 1 in the Supporting Information); which in some cases produced separate quantum-wire specimens having the same diameters. 

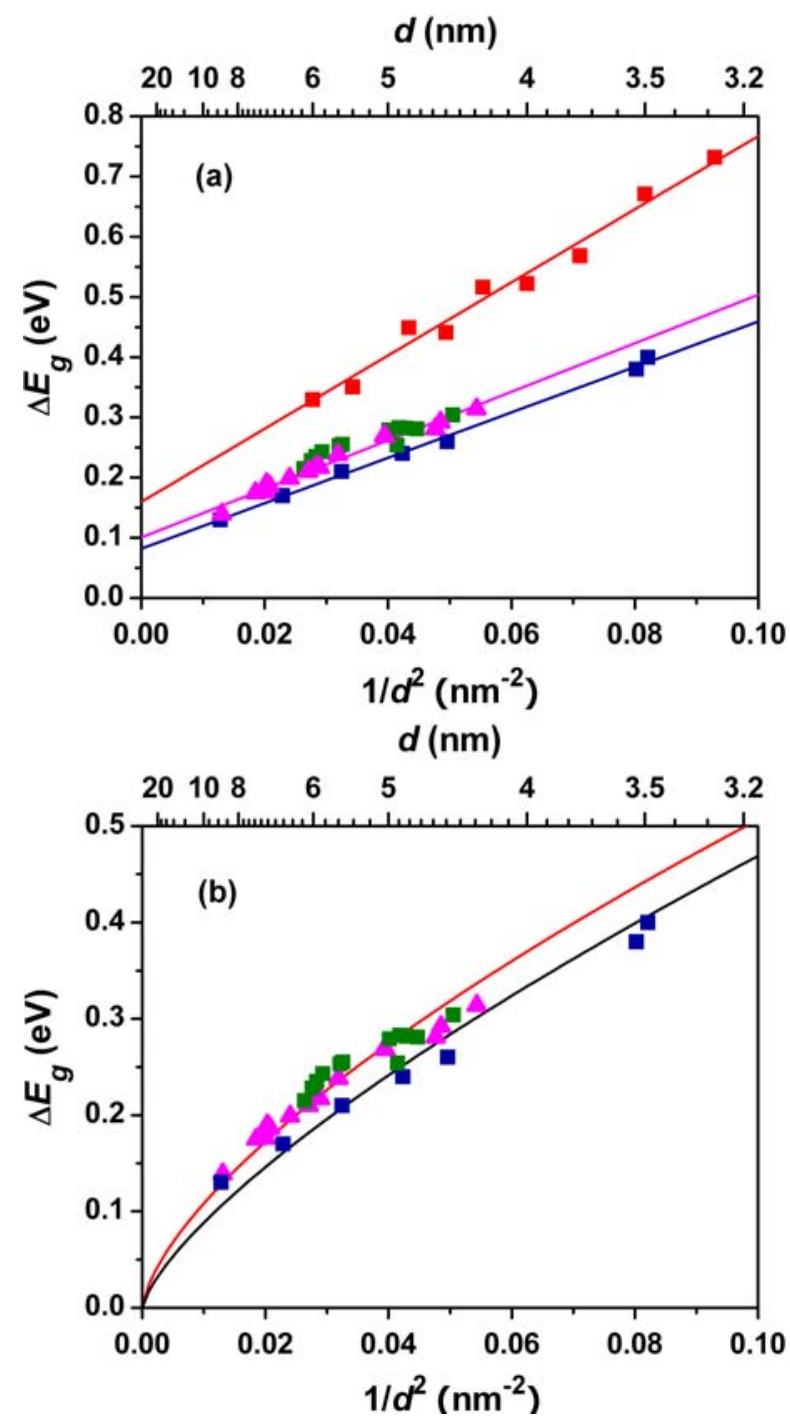

Figure 3. Experimental band-gap data measured from the absorption spectra of InP quantum dots (red squares) ${ }^{2,25,26}$ and quantum wires (pink triangles, HDA/TOPO/TOP-surfactant passivated; green squares, HF thinned; blue squares, polymer passivated $\left.{ }^{14}\right)$, plotted as $\Delta E_{\mathrm{g}}$ vs. $1 / d^{2}$. (a) The red, pink, and blue lines are linear least squares fits to the dot and wire data, respectively. The slopes are $A_{\mathrm{dot}}=6.1 \pm 0.1$ $\mathrm{eV} \mathrm{nm}^{2}, A_{\text {wire-surfactants }}=4.0 \pm 0.2 \mathrm{eV} \mathrm{nm}^{2}$, and $A_{\text {wire-polymer }}=3.8 \pm 0.2 \mathrm{eV} \mathrm{nm}^{2}$; and (b) The orange and black curves are the theoretical predictions for the diameter-dependence of band-gap energies for InP quantum wires based on DFT-LDA-CPM $\left(\Delta E_{\mathrm{g}}=2.37 d^{-1.34}\right)^{29}$ and SEPM $\left(\Delta E_{\mathrm{g}}=2.49 d^{-1.45}\right)^{14}$ calculations, respectively. The experimental data (points) are the same as those in (a). 

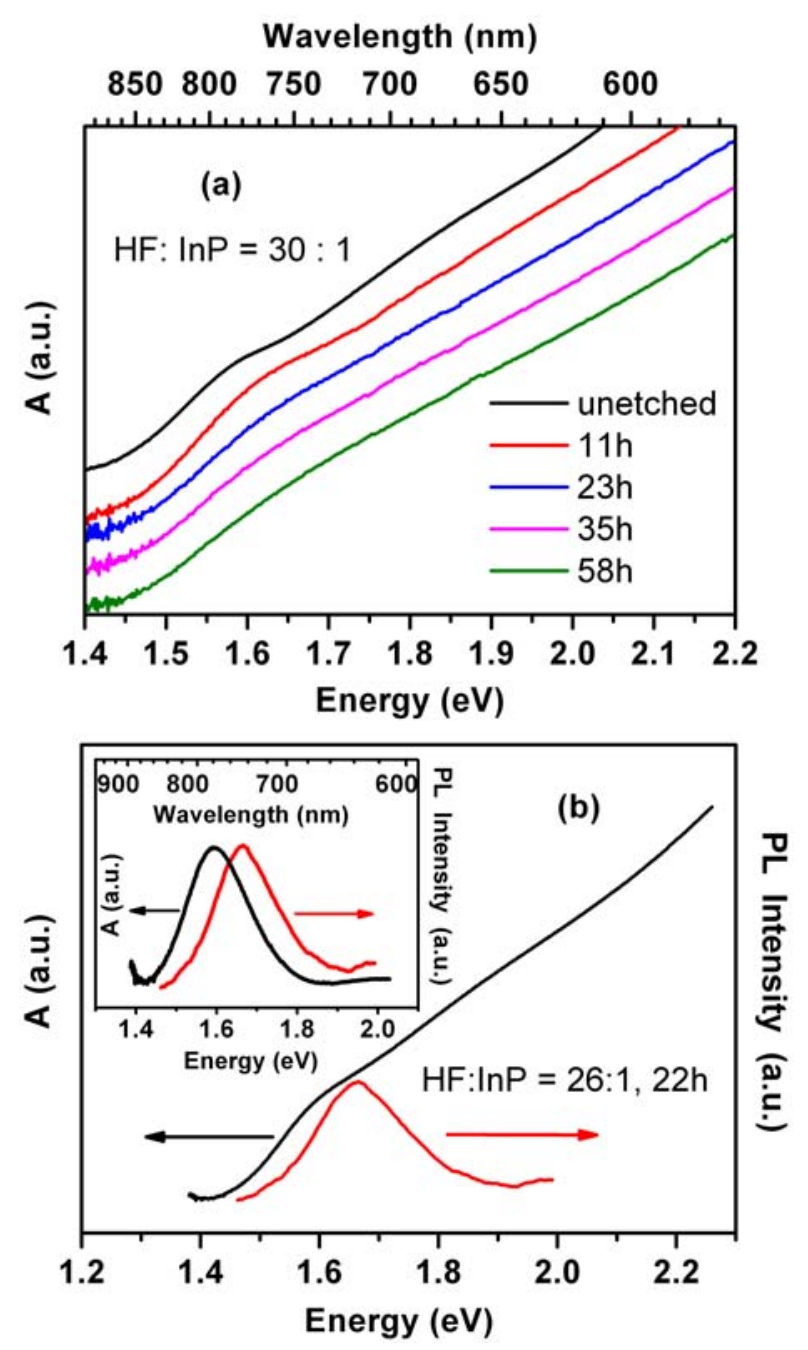

Figure 4. Absorption spectra of HF-photoetched InP wires under the filtered xenon irradiation. (a) Absorption spectra at different etching times with $\mathrm{HF} / \mathrm{InP}=30 / 1$, showing the gradual disappearance of the excitonic feature; and (b) Representative absorption and PL spectra when a low HF/InP ratio (26/1) and short photoetching period was used. The black feature in the inset is the lowest-energy excitonic peak from the absorption spectrum extracted by nonlinear least-squares fitting and background subtraction. The PL feature (red) is blue shifted about $0.1 \mathrm{eV}$ from the absorption feature (black). 

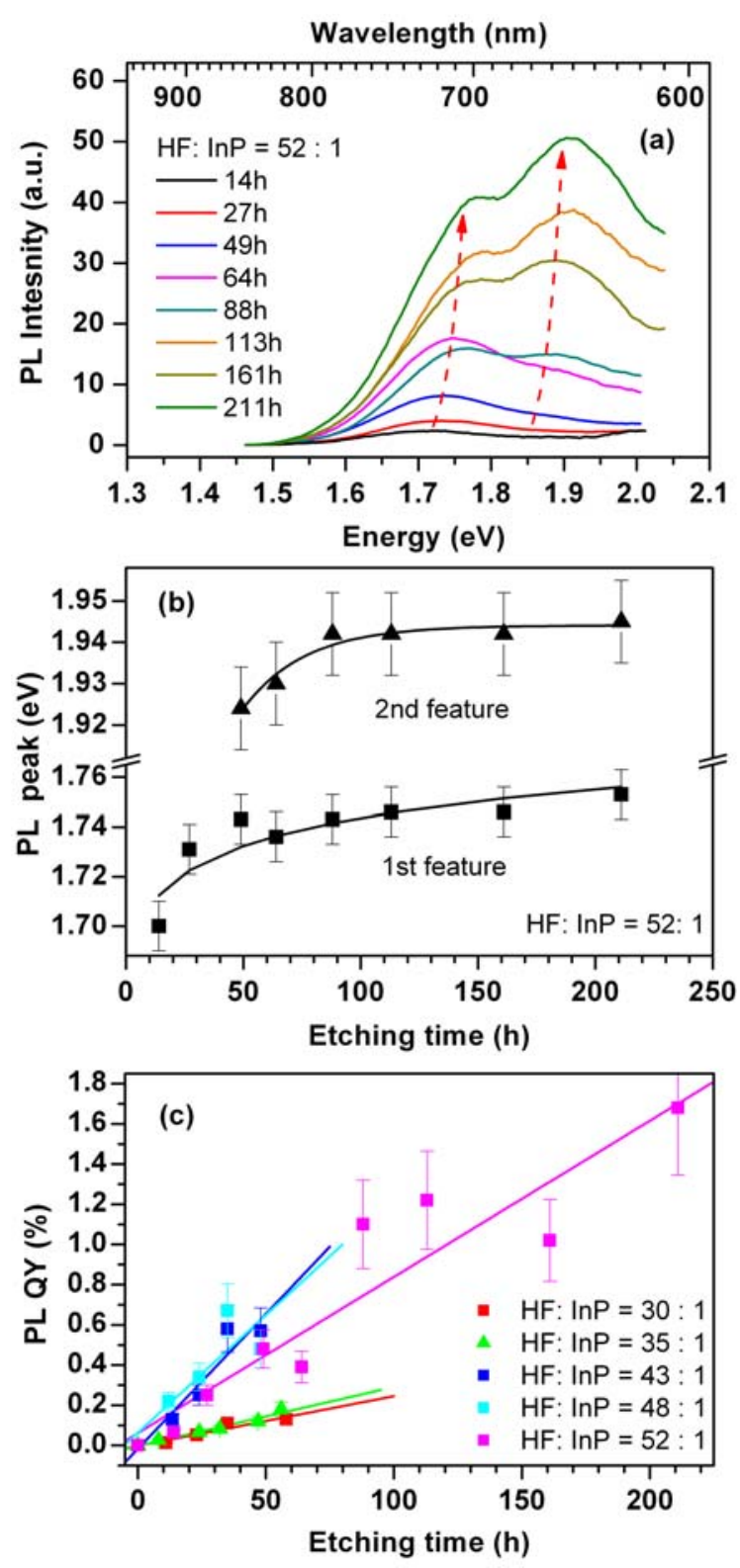

Figure 5. HF-photoetching of InP wires under a filtered xenon lamp ( $\lambda>700 \mathrm{~nm})$. (a) PL spectra at different etching times with $\mathrm{HF} / \mathrm{InP}=52 / 1$. The red dashed arrows are guides to the eye; (b) Etchingtime dependence of PL-peak energy shift $(\mathrm{HF} / \mathrm{InP}=52 / 1)$. The black curves are guides to the eye; and (c) Effective PL quantum yield as a function of etching time at various HF/InP ratios. The lines are linear least-squares fits to guide the eye. 


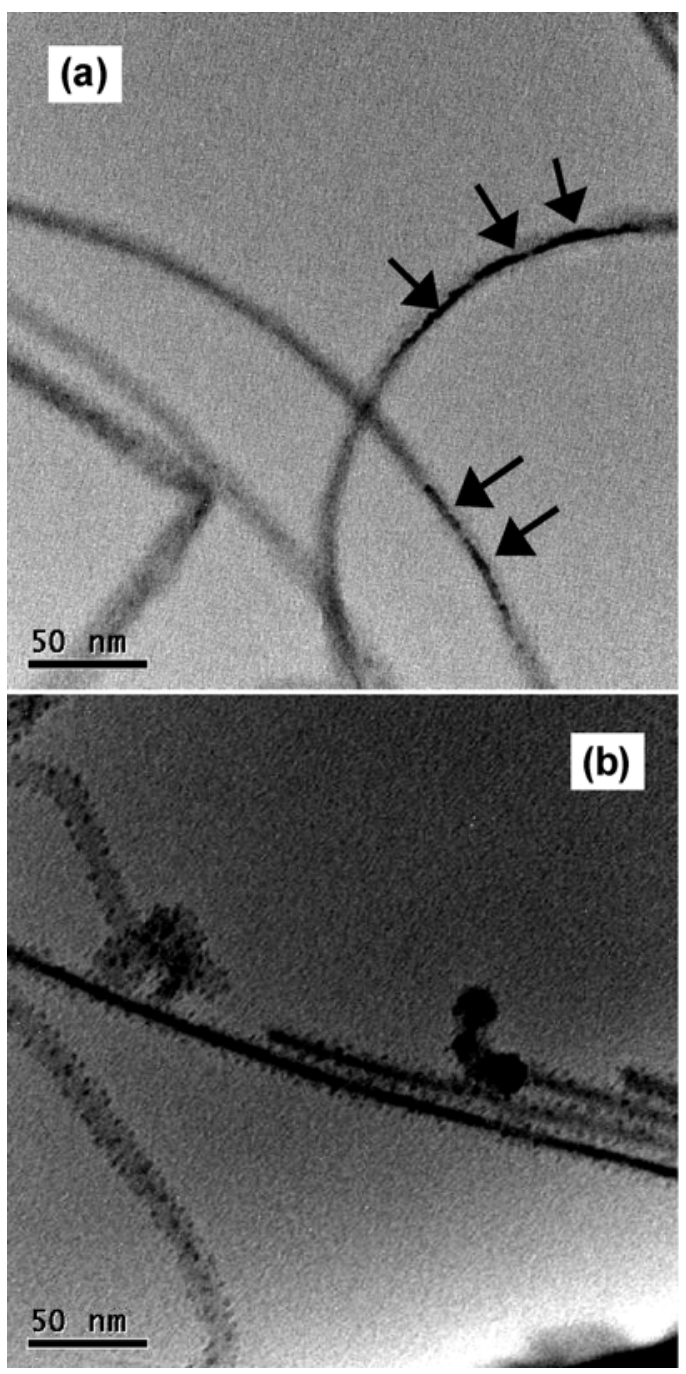

Figure 6. Representative TEM images of InP wires HF-photoetched under filtered xenon irradiation. (a) The arrows identify high-contrast crystalline InP domains, which are mainly thin rod-like structures. The remaining areas of the wires with low contrast are mainly amorphous with diameters much larger than the initial values; and (b) Small crystalline InP domains consisting of dots with diameters of ca. 3 $\mathrm{nm}$ decorating amorphous wire regions (light contrast) and remaining crystalline InP wires (dark contrast). 


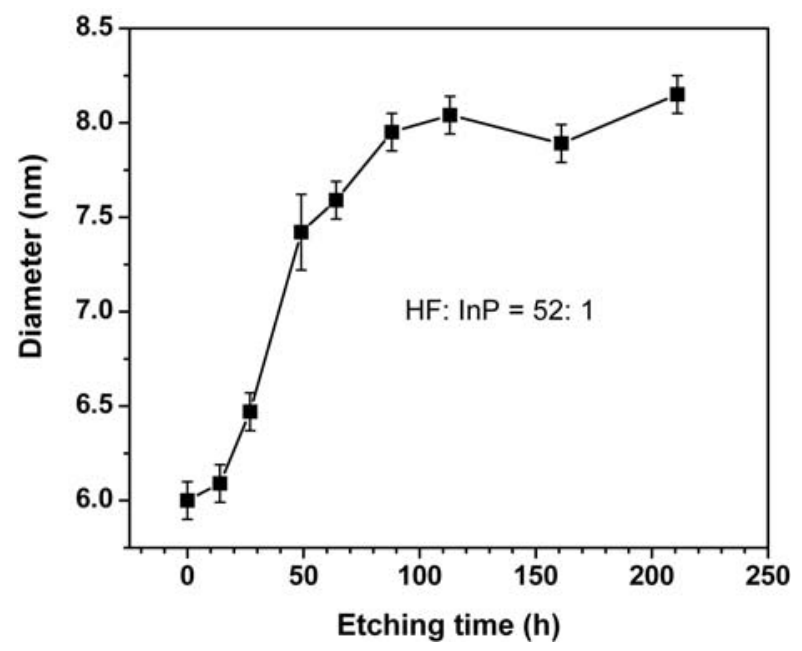

Figure 7. Plot of TEM wire diameter as a function of etching time $(\mathrm{HF} / \mathrm{InP}=52 / 1)$. The black curve is a guide to the eye. The error bars are the difference between the statistical mean diameters and the peaks of the Gaussian fits to the diameter distributions (see Supporting Information and Figure S13 for details). 

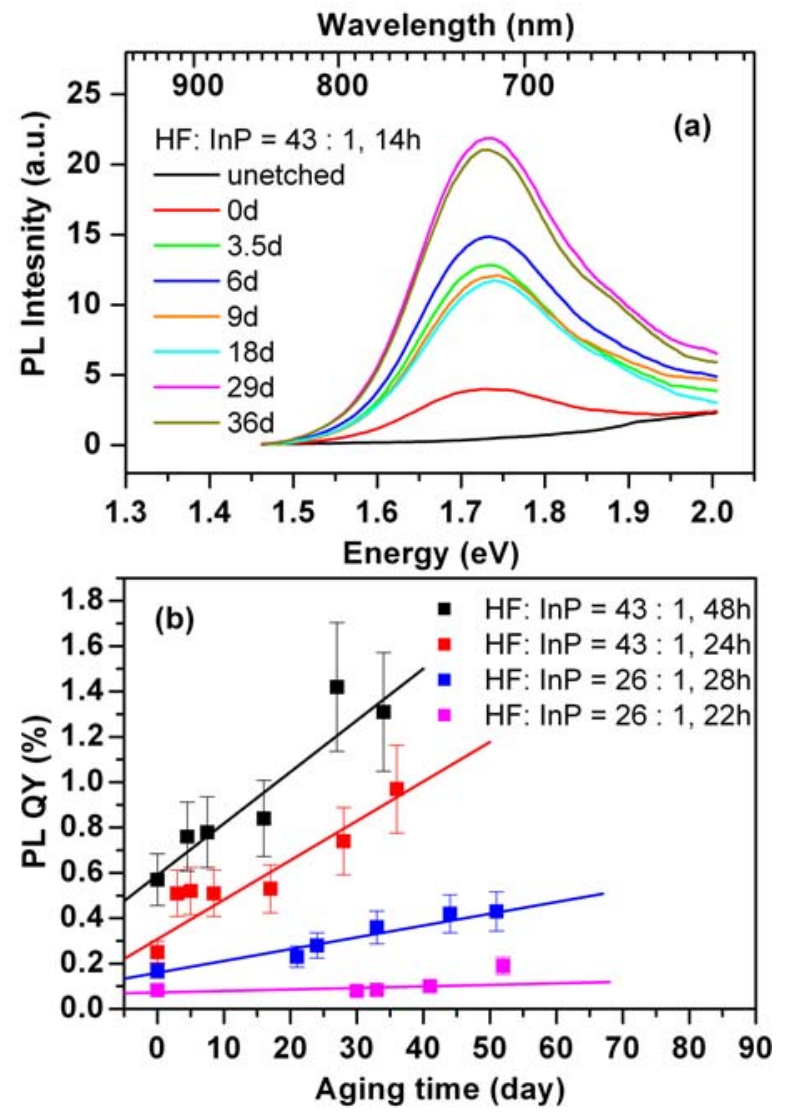

Figure 8. Photo-aging of HF-photoetched InP wires under room light. (a) PL spectra at different aging times after $14 \mathrm{~h}$ of photoetching with $\mathrm{HF} / \mathrm{InP}=43 / 1$; and (b) Effective PL quantum yield as a function of aging time. The legend shows the HF/InP ratios and etching times. The lines are linear least-squares fits to guide the eye. 

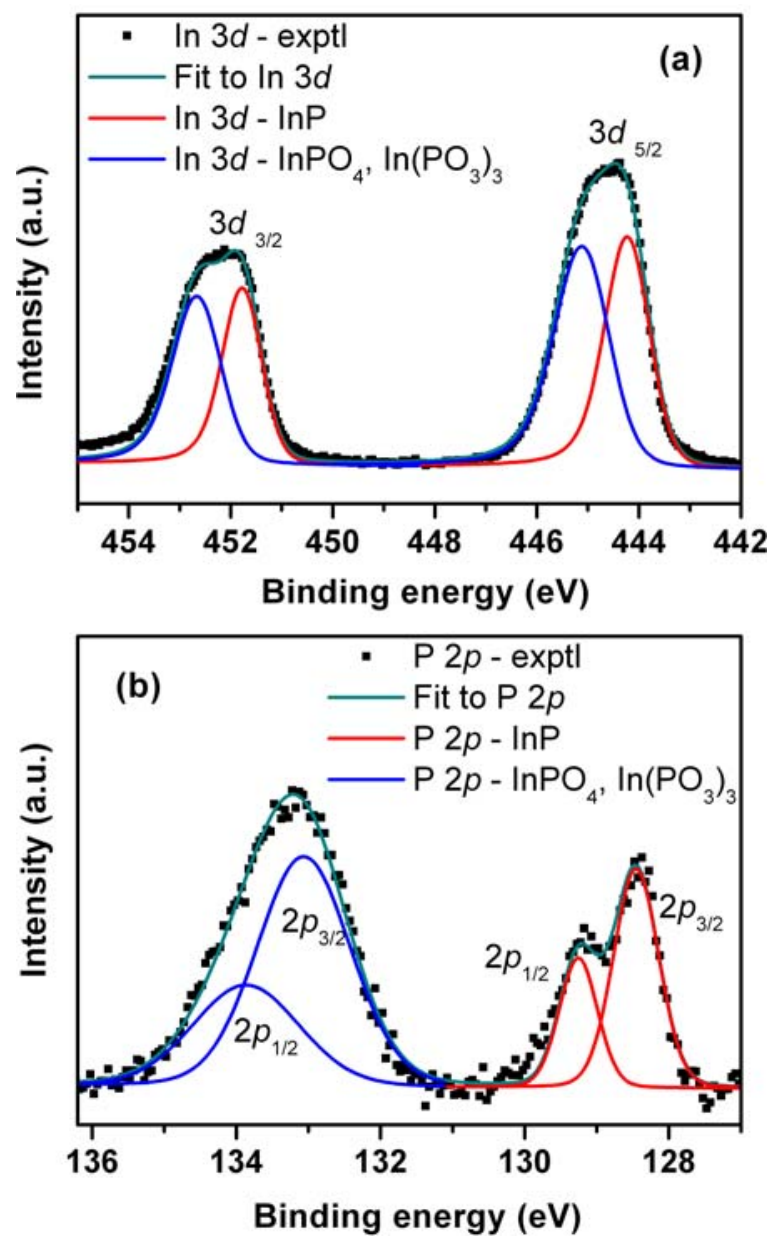

Figure 9. XPS from HF-photoetched InP wires $(\mathrm{HF} / \mathrm{InP}=52 / 1$, etching time $=120 \mathrm{~h}$ under filtered xenon irradiation). (a) In $3 d$ core level; and (b) P2p core level. Binding energies and FWHM are listed in Table 4 in the Supporting Information. 


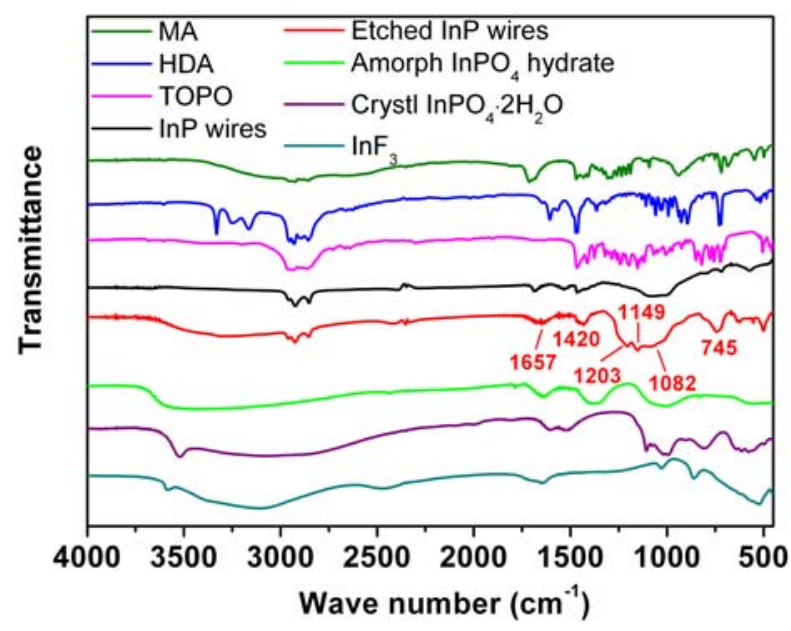

Figure 10. FTIR spectra for unetched (black) and HF-photoetched InP wires (red, HF/InP $=52 / 1$, etching time $=88 \mathrm{~h}$ under filtered xenon irradiation). The spectra for myristic acid (MA, olive), HDA (blue), TOPO (pink), amorphous $\mathrm{InPO}_{4}$ hydrate (green), crystalline $\mathrm{InPO}_{4} \cdot 2 \mathrm{H}_{2} \mathrm{O}$ (purple), and $\mathrm{InF}_{3}($ dark cyan) were added for comparison. 
Table of Contents (TOC) Graphic

\section{Spectroscopic Properties of Colloidal Indium Phosphide Quantum Wires}

Fudong Wang, Heng Yu, Jingbo Li, Qingling Hang, Dmitry Zemlyanov, Patrick C. Gibbons, Lin-Wang Wang, David B. Janes, and William E. Buhro
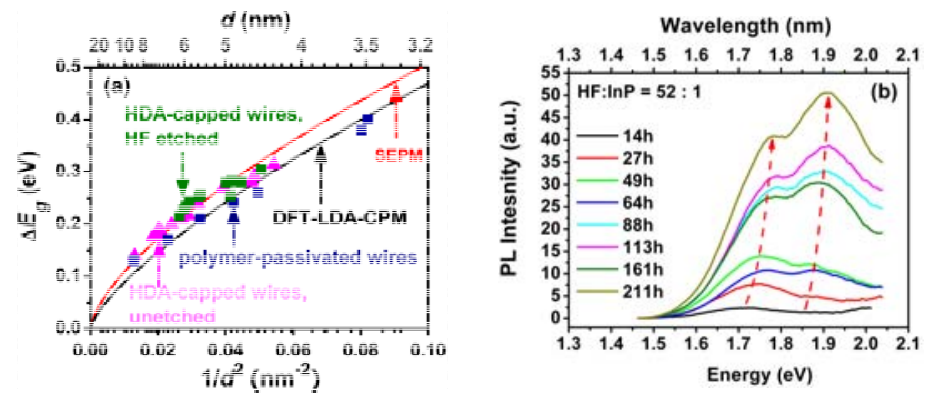